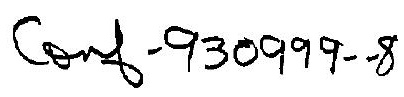

UCRL-JC-115916

PREPRINT

\title{
Equilibration and Multifragmentation in Heavy Ion Reactions
}

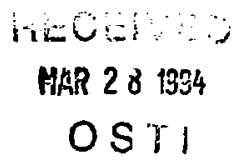

M. G. Mustafa

M. Blann

A. Botvina

This paper was prepared for submittal to the

NATO Advanced Study Institute on Hot and Dense Nuclear Matter

Bodrum, Turkey

September 26-October 9, 1993

September, 1993

This is a preprint of a paper intended for publication in a joumal or proceedinge. Since changes may be made bafore publiation, this preprint is made available with the understanding that it will not be cited or reprodused without the permission of the apthor. 


\section{DISCLAIMER}

This doeument was prepared es an account of work ppowered by an atgency of the Uniled Sute Governenet Nether the Unied Stetes Government wor the Univertity of Cilifornia nor any of their enfloyees, makes any warreoty, expess or inplied, or

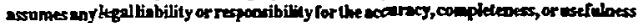
of any inform stion, spparates, product, or process fiedoed, or nepresents that its use

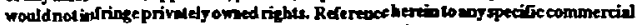
produrts, process, or service by trode nene, traderark, movefacturer, or atherwise,

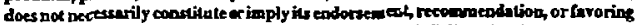
by the United Steres Goremmens of the University of Cariformin The views nod apinions of anchos empreseed herein da not necesserif state or relled those of the United Sutes Governenent or the University of Cdifomin, and thall not be nsed for edvertising or product endorrenent purposes. 


\title{
EQUILIBRATION AND MULTIFRAGMENTATION IN HEAVY ION REACTIONS
}

\author{
G. Peilert, ${ }^{1}$ M. G. Mustafa, ${ }^{1}$ M. Blann, ${ }^{1}$ and A. Botvina ${ }^{2}$ \\ 1 Lawrence Livermore National Laboratory \\ Livermore, $\mathrm{CA} 94550$ \\ 2 Institute for Nuclear Research, Russian Academy of Sciences, \\ 117312 Moscow, Russia
}

\section{INTRODUCTION}

Modeling of multifragmentation measurements from heavy ion reactions generally requires separate treatment of the initial fast part of the reaction, during which energetic nucleons are emitted, and of a quasi-equilibrated system where sufficient degrees of freedom have been excitod, so that statistical approaches may be applied. Some of the more sophisticated fast cascade models, e.g., Quantum molecular dynamics (QMD), might also produce fragment yields, however, transport models have not yet been able to satisfactorily reproduce fragmentation properties of nuclear reactions.

In this work we consider the interactions of $36 \mathrm{Ar}$ with $197 \mathrm{Au}$ at incident energies of 35 to $110 \mathrm{MeV} \cdot \mathrm{A}$, which was investigated by de Souza et al. ${ }^{1}$ We will first look at two dynamic models which may be used to estinate the excitation remaining for quasi-equilibrated systems following the fast nucleonic cascade, specifically the Boltzmann master equation (BME) 2-5 and Quantum molecular dynamics (QMD) approaches. 6,7 Using excitations from the BME model to estimate values for quasi-equilibrated nuclei, we will explore two approaches to statistical multifragmentation calculations, one of sequential binary decay, ${ }^{8-10}$ the other a simultaneous multifragmentation model (SMM).11-13 We will consider central collisions in our calculations, and experimental results gaied on the highest total charged particle emissions - those deduced to be central collisions.

\section{EQUILIBRATION (FAST CASCADE) MODELS}

The QMD model is well known, and is still being improved for applications in relativistic heavy ion reactions. We refer to published references for details. 6,7 
In this work we will show results with a form of 'Pauli potential'. The BME, unlike many models, follows reactions in energy space under the assumption that the geometric aspects - nucleon directions - roughly average out in the many collisions involving incident beam velocities and random Fermi velocities, and that we may make use of the well known isotropic energy distribution in nucieonnucleon scattering.

The method of dynamics in the BME for heavy ion reactions is as follows: as the nuclei interact, we assume that the projectile (lighter partner) puts nucleons into the well of the heavier partner which mainly determines the center of mass of the reaction. We assume that every energy conserving partition is equally likely due to coupling of Fermi and beam momenta. In this case an exciton state density expression may be used to partition the energy. 5

The BME ther follows the rate of nucleon-nucleon scattering, (using energy and isospin dependent cross sections) in competition with an emission rate for the population in each energy bin. 2,3 Due to the simplicity of the model, the time dependent properties of many variables are easily followed for the two component gas, and even for the recoil momenta of the residues once a parameterization for emitted nucleon angular distributions is selected. ${ }^{14}$ The time step in the BME may be varied; by default we use time increments of $2 \times 10^{-23} \mathrm{sec}$, usually following $50-$ 100 steps.

\section{Model Intercomparisons}

In Figures 1 and 2 we compare the time dependence of the QMD and BME models at incident energies of 35,80 and $110 \mathrm{MeV} \cdot \mathrm{A}$ for ${ }^{36} \mathrm{Ar}$ on ${ }^{197} \mathrm{Au}$. Due to dimension limits in the $\mathrm{BME}, 100 \mathrm{MeV} \cdot \mathrm{A}$ was used rather than 110 . We show the $\mathrm{E}_{\mathrm{CM}}+\mathrm{Q}$ for each reaction as a horizontal bar; this would be the excitation of a compound nucleus if one were formed.

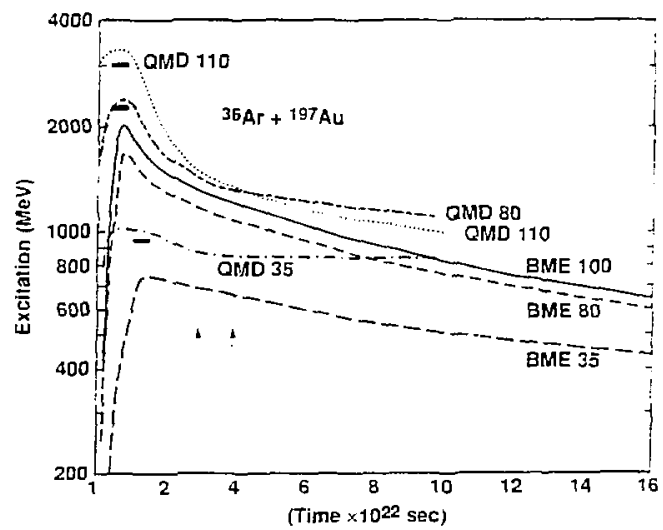

Figure 1. Time dependence of total excitation versus time for the interaction of 35,80 and $110 \mathrm{MeV} \cdot \mathrm{A}{ }^{36} \mathrm{Ar}$ with ${ }^{197} \mathrm{Au}$. The horizontal bars indicate the $\mathrm{ECM}+\mathrm{Q}$ for each reaction. The vertical arrows at 3 and $4 \times 10^{-22} \mathrm{sec}$ indicate the times at which excitations were taken for subsequent equilibrium calculations. 


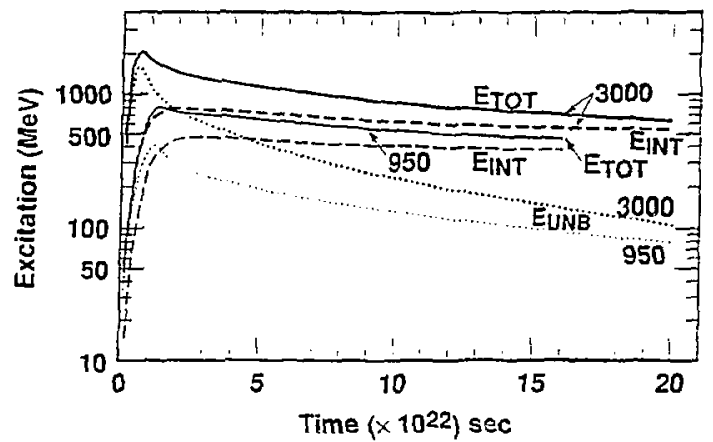

Figure 2. Time dependence of excitation for 35 and $110 \mathrm{MeV} \cdot \mathrm{A} 36 \mathrm{Ar}+{ }^{197} \mathrm{Au}$ reactions, according to the BME model. The reactions are designated by corresponding $\mathrm{E}_{C \mathrm{M}}+\mathrm{Q}$ values, 950 and 3000 respectively. The total excitation versus time, as shown in Figure 1, has been divided into particle (and hole) energy of quasi-bound nucleons $\left(E_{i n t}\right)$ and energy carried by neutrons above the binding energy plus protons above the binding plus Coulomb energies (EUNB).

In the $B M E$, we see that the $E_{C M}+Q$ is never available to the two interacting nuclej; this is because nucleon emission during coalescence removes a substantial part of the reaction energy. The higher the incident energy per nucleon, the larger the fraction removed, making it increasingly difficult to gain high internal excitation. The QMD has qualitative similarities, but at these relatively low incident energies some of the longer time behavior is perhaps open to large dependencies on details of the calculation which are still under development.

Arrows in Figure 1 show times of 3 and $4 \times 10^{-22} \mathrm{sec}$; typically we judge systems to be quasi-equilibrated within that time range. The greater the incident energy, the shorter the equilibration period.

Figure 2 shows some more detailed aspects of the $100 \mathrm{MeV} \cdot \mathrm{A}$ and $35 \mathrm{MeV} \cdot \mathrm{A}$ systems. We show the total excitation, as on the previous figure. We also show the energy of unbound nucleons (above the Coulomb barrier for protons) and of bound nucleons plus holes. The unbound nucleons will have a large probability of emission in a single transit of the nuclear diameter, while the bound excitation nucleons and holes - have a much higner probability of undergoing multiple collisions and producing a quasi-equilibrated system. We see in Figure 2 that the internal (bound/quasi-bound) energy exceeds the unbound energy for the two cases shown at around $2 \times 10^{-23} \mathrm{sec}$. We also note that the unbound energy monotonically decreases after this time, as does the total excitation, rendering the question of the 'time' and therefore the excitation of the 'equilibrated' system a subjective issue. We will show the sensitivity of multifragmentation yields to excitation in a few cases to be presented; first we show a few further comparisons of the BME and QMD approaches.

In Figures 3 and 4 we compare emitted nucleon multiplicity vs. time at 35 and $110 \mathrm{MeV} \cdot \mathrm{A}$. Qualitatively at $110 \mathrm{MeV} \bullet \mathrm{A}$ the results are similar, but many more nucleons are emitted in the QMD than in the BME model. At $35 \mathrm{MeV} \cdot \mathrm{A}$ the same is true up to about $90 \mathrm{fm} / \mathrm{c}\left(3 \times 10^{-22} \mathrm{sec}\right)$, after which emission in the QMD model suddeniy drops to a very low instantaneous value. 


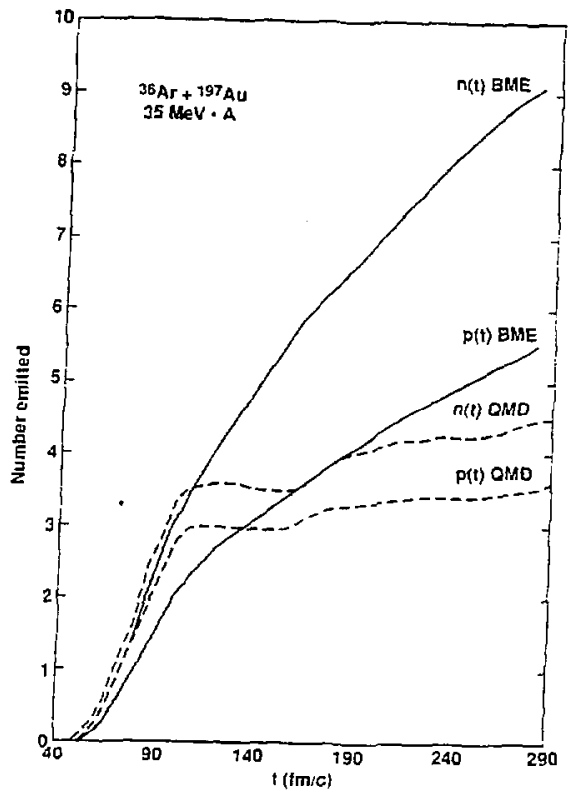

Figure 3. Emilted neutron multiplicity versus time in the BME and QMD models for $35 \mathrm{MeV} \cdot \mathrm{A}$ $36 \mathrm{Ar}+{ }^{197} \mathrm{Au}$. The vaiues shown are the integrals up to time ' $\mathrm{t}$ '.

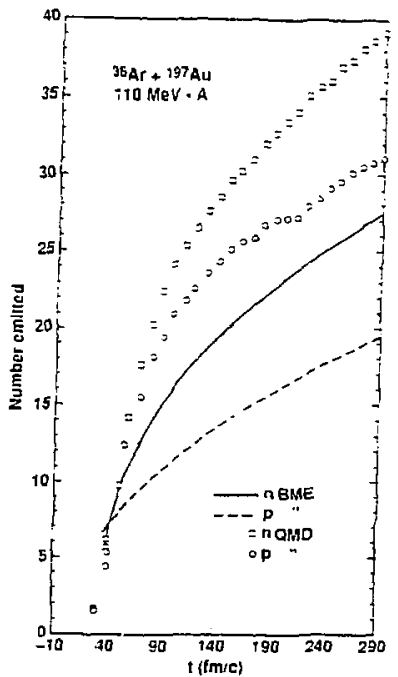

Figure 4. As in Figure 3 for $110 \mathrm{MeV} \cdot \mathrm{A}$ incident energy. 
The relationship of kinetic energies between the two models is displayed in Figures 5 and 6 . For the BME the lower curves show the instantaneous kinetic energy per time step (Figure 6); all other points and curves are the resulis averaged up to time ' $t$ '. The iwo models are qualitatively similar, the greatest difference being in the Coulomb boost observed for proton kinetic energies in the BME which is not reflected in the QMD results.

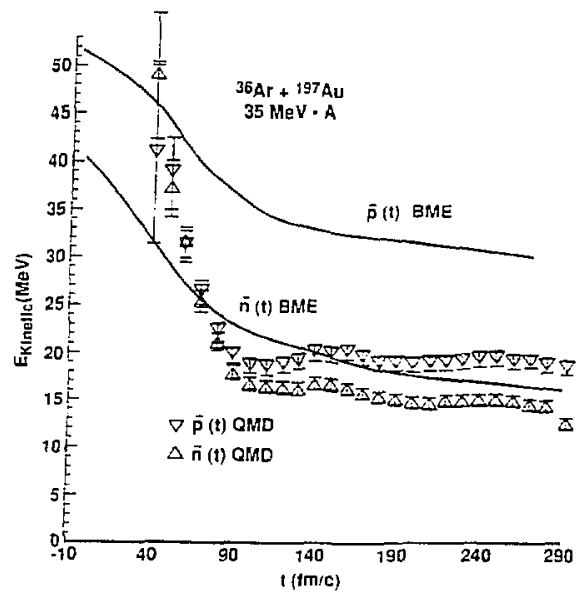

Figure 5. Kinetic energy of emitted neutrons and protons versus time in the BME and QMD approaches. The emission energies represent the kinetic onergy of the neutron (proton) averaged up to the time ' $t$ ' shown on the abscissa. Incident ${ }^{36} \mathrm{Ar}$ energy was $35 \mathrm{MeV} \cdot \mathrm{A}$.

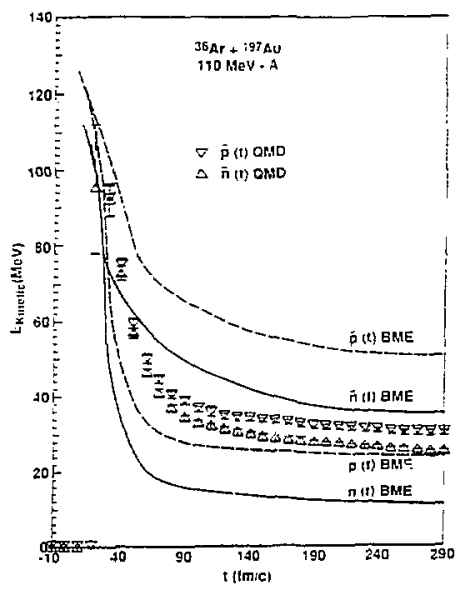

Figure 6. Kinetic energy of emitted neutrons and protons versus time as predinted in the BME and QMD models. These results are for $110 \mathrm{MeV} \cdot \mathrm{A} 36 \mathrm{Ar}$ on a $197 \mathrm{Au}$ target. Results are for kinetic energies averaged up to time ' $t$ '; for the BME model we also show the kinetic energies of emission at time ' $t$ ', $(p(t), n(t))$. 
We have seen that there are uncertainties and ambiguities as to how we extract an 'excitation at equilibrium' following the fast cascade - which becomes monotonically lower with time in a continuous fashion. We have used the BME results at $4 \times 10^{-23} \mathrm{sec}$ for calculating the exclusive multiplicities for ${ }^{36} \mathrm{Ar}+{ }^{197} \mathrm{Au}$ at $35,50,80$ and $100 \mathrm{MeV} \cdot \mathrm{A}$; for 80 and $110 \mathrm{MeV} \cdot \mathrm{A}$ we also calculated results for successive binary decay using the excitation at $3 \times 10^{-22} \mathrm{sec}$, in order to demonstrate the sensitivity of final results to the assumption of excitation remaining in an 'equilibrated' system.

\section{Multifragmentation Yields}

In Figure 7 we show the experimental results of de Souza et.al. 1 for incident energies between 35 and $110 \mathrm{MeV} \cdot \mathrm{A}$. We show results for gates set on either the highest toidl charged particle multiplicities, or the highest two multiplicities. These presumably represent the more central collisions, corresponding to those used for the BME and QMD models. The SMM considers the partition of the equilibrated system into all possible fragments as to mass and multiplicities, based on a liquid drnp mass formula and thermal excitation of the equilibrated system. The hot fragments are then allowed to de-excite to ground via a simple evaporation model. The iragmenting nucleus is assumed to come from a low density nucleon gas; for this work a density of half saturation value was assumed.

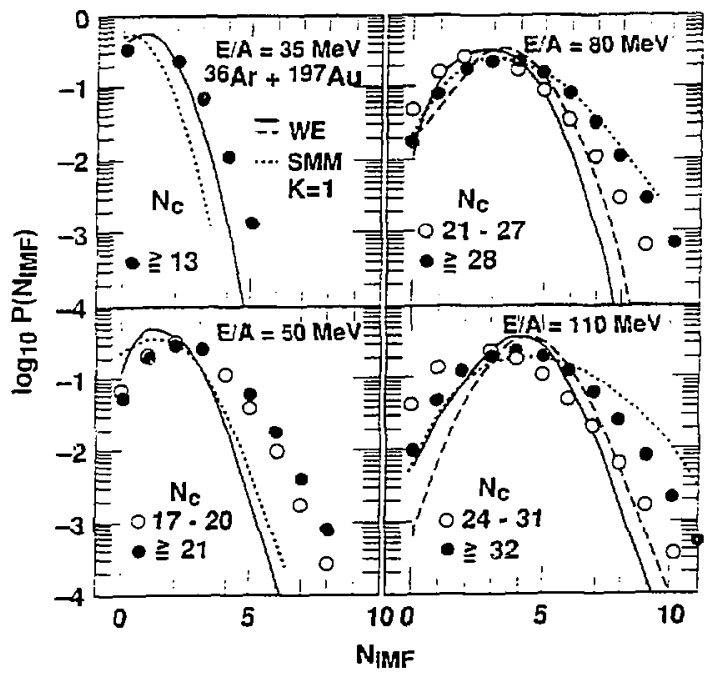

Figure 7. Experimental and calculated fragmentation multiplicities for $35,50,80$ and $100 \mathrm{MeV} \cdot \mathrm{A}$ $36 \mathrm{Ar}+{ }^{197} \mathrm{Au}$. The experimental results given by open and closed circles are those of de Souza et al. The total charged particle multiplicity gates used are indicated. Results using excitations from the BME at $4 \times 10^{-22} \mathrm{sec}$ are shown by solid lines for the sequential binary decay result (for primary fragments) and by dotted lines for the statistical (simultaneous) multifragmentation model. The dashed curves at 80 and $100 \mathrm{MeV}$. A are the sequential binary model resuits using excitations at $3 x$ $10^{-23}$ sec. 
For the statistical calculations presented in Figure 7 for incident 36 Ar energies of $35,50,80$ and $110 \mathrm{MeV} \cdot \mathrm{A}$ with $\mathrm{ECM}+\mathrm{Q}$ values of 952, 1409, 2322 and $2950 \mathrm{MeV}$, we used the BME predicted total excitations of 651,827, 1076 and $1200 \mathrm{MeV}$, respectively at $4 \times 10^{-22} \mathrm{sec}$ after contact. The number of emitted neutrons and protons, used to deduce the equilibrated nuclei, were respectively $5.9 \mathrm{n}, 3.7 \mathrm{p} ; 9.2 \mathrm{n}, 6.0 \mathrm{p} ; 14.9 \mathrm{n}, 10.2 \mathrm{p}$; and $18.3 \mathrm{n}, 12.8 \mathrm{p}$. At 80 and $110 \mathrm{MeV} \bullet \mathrm{A}$ incident energy we also produce results for sequential binary decay assuming equilibration takes place at $3 \times 10^{-22} \mathrm{sec}$; for these cases the excitations were 1171 and $1320 \mathrm{MeV}$ respectively, with $12.5 n, 8.7 p$ and $15.5 n, 10.9 p$ being emitted.

The Weisskopf-Ewing (W-E) model has been extended in the ALICE code to include level densities for the emitted fragments, ${ }^{9}$ with a sum over all partitiuns of internal excitation and channel energy between light and heavy fragments. The ALICE code was modified both to include emission of up to 20 clusters (up to ${ }^{48} \mathrm{Ca}$ ), and to outfut the internal spectral excitations on an exciusive basis, i.e., clusters coming from a nucleus which had emitted no clusters before, one cluster before, etc. 9,10 However, at the higher excitations of Figure 7 (higher incident ${ }^{36} \mathrm{Ar}$ energies), some fraction of the clusters may themselves emit clusters in decaying to ground, while others will decay by nucleon and alpha emission to non-cluster residues. These decays will somewhat broaden calculated primary distributions, but they are not easily treated in an exclusive mode in the ALICE code. We therefore present primary distributions for the sequential binary decay (W-E) case in Figure 7.

The $S^{\prime} "$ " $i$ curves in Figure 7 are the successive binary results using excitations from the BME at $4 \times 10^{-23} \mathrm{sec}$ after first contact, as were used in the SMM calculations. The dashed curves result from using the excitations at $3 \times 10^{-23} \mathrm{sec}$ after contact. The two models (W-E and SMM) are rather close in their predictions, particularly at the two lower energies. At the higher energies the SMM results are in somewhat better agreement with the data; however, the W-E results may be expected to broaden upon de-excitation to ground. This would bring about a closer agreement between the two models. 
The BME and QMD formulations give qualitatively similar behaviors for relaxation during heavy ion collisions. Results differ on a quantitative basis. Systems progress monotonically toward equilibrium following initiation of a reaction; the selection of a time, and therefore the excitation of an equilibrated system is subjective. This puts an uncertainty on the input into any equilibrium model. In comparing a sequential binary model with a simultaneous multifragmentation model, we get results which are reasonably consistent within the uncertainties of the choice of initial excitation, and effects of decay of primary fragments in the sequential binary model. Selection between models will therefore require tests other than exclusive multiplicities.

This work was performed under the auspices of the U.S. Department of Energy by the Lawrence Livermore National Laboratory under contract number W-7405. ENG-48. 


\section{REFERENCES}

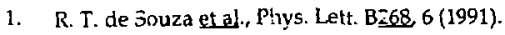

2. C. D. Harp, ]. I. Miller, and B. J. Berne, Phys. Kev. 165 1166 (19u8).

3. G. D. Harp and J. M. Miller, Phys. Rev. C3, 1847 (1971).

4. M. Blann, Nucl. l'hys. A235 211 (1974).

5. M. Blann. Phys. Rev. C31 1245 (1985).

6. G. Peilert, H. Stöcker, W. Greiner, A. Rosenhauer, A. Bohnet, I. Aichelin: Mod. Phys. Lett. A3, 459 \{1988); Phys. Rev. C39 1402 (2989).

7. J. Aichelin, G. Peilert, A. Bohnet, A. Rosenhauer, H. Stöcker, W. Greiner: I'hys. Rev. C3Z 2451 (1988).

8. M. Biann, T. Korroto, and 1. Tserruya, Phys. Rev. C40, 2498 (1989).

9. M. Blann and M. G. Mustaia, Phys. Rev. C44, R590 (1991).

10. A. Botvina, I. N. Mishustin, M. Blann, M, G. Mustafa, G. Peilert, H. Stöcker, and W. Greint r: Z. Phỵsik A335 297 (1993).

11. A. S. Botvina, A. S. Iljinov, I. N. Mishustin: Sov. J. Nucl. Phys. 42,712 (1985).

12. A. S. Botvina, A. S. Iljinov, I. N. Mishustin, J. P. Bondorf, R. Donangelo, K. Sneppen: Nucl. Phys. A475, 663 (1987).

13. A. S. Bot .na, A. S. Iljinov, I. N. M:shustin: Nucl. Phys. A507, f.x (1990).

14. M. Blann, Phys. Rev. C3\ 295 (1985). 